\title{
A Poor Prognostic Metastatic Nongestational Choriocarcinoma of the Ovary: A Case Report and the Literature Review.
}

\section{Kimihiro Nishino ( $\square$ kimikimi@med.nagoya-u.ac.jp )}

Nagoya University Graduate School of Medicine Faculty of Medicine: Nagoya Daigaku Daigakuin Igakukei Kenkyuka Igakubu https://orcid.org/0000-0002-2228-8450

\section{Eiko Yamamoto}

Nagoya University Graduate School of Medicine Faculty of Medicine: Nagoya Daigaku Daigakuin Igakukei Kenkyuka Igakubu

\section{Yoshiki lkeda}

Nagoya University Graduate School of Medicine Faculty of Medicine: Nagoya Daigaku Daigakuin Igakukei Kenkyuka Igakubu

\section{Kaoru Niimi}

Nagoya University Graduate School of Medicine Faculty of Medicine: Nagoya Daigaku Daigakuin Igakukei Kenkyuka Igakubu

\section{Toshimichi Yamamoto}

Nagoya University Graduate School of Medicine Faculty of Medicine: Nagoya Daigaku Daigakuin Igakukei Kenkyuka Igakubu

Hiroaki Kajiyama

Nagoya University Graduate School of Medicine Faculty of Medicine: Nagoya Daigaku Daigakuin Igakukei Kenkyuka Igakubu

\section{Case report}

Keywords: pure ovarian nongestational choriocarcinoma, short tandem repeat analysis, BEP therapy

Posted Date: October 16th, 2020

DOI: https://doi.org/10.21203/rs.3.rs-90334/v1

License: (c) (i) This work is licensed under a Creative Commons Attribution 4.0 International License. Read Full License

Version of Record: A version of this preprint was published at Journal of Ovarian Research on April 22nd, 2021. See the published version at https://doi.org/10.1186/s13048-021-00810-3. 


\section{Abstract}

Background: Pure ovarian choriocarcinoma can be gestational or nongestational in origin. Nongestational pure ovarian choriocarcinoma is extremely rare and the prognosis is thought to be worse than that of the gestational type in patients with metastatic disease. We present a case of metastatic pure ovarian choriocarcinoma with poor prognosis in which the origin was identified as nongestational by DNA short tandem repeat (STR) analysis.

Case presentation: A nulliparous woman in her thirties with metastatic choriocarcinoma was referred to our hospital after initial treatment proved unsuccessful. Two months earlier, she had undergone brain tumor resection and histological examination confirmed choriocarcinoma. Serum human chorionic gonadotropin (hCG) concentration at initial diagnosis was $5030 \mathrm{IU} / \mathrm{L}$. Two cycles of a combination chemotherapy regimen of methotrexate, etoposide, and actinomycin-D (MEA therapy), which is commonly used for gestational choriocarcinoma, was administered. However, the disease could not be controlled. Imaging modalities at presentation revealed tumor present in the left ovary and left lung, but not in the uterus, which led us think that the choriocarcinoma was nongestational. Bleomycin, etoposide, and cisplatin (BEP therapy) which is commonly used for nongestational choriocarcinoma (malignant germ cell tumor) and surgical resection of the uterus, bilateral ovaries, and an affected part of the left lung led to the nadir level of hCG, but the tumor relapsed and levels of hCG again increased. To investigate the origin of choriocarcinoma, we performed DNA STR analysis of tumor cells and oral mucosal cells. Analysis revealed the origin of the choriocarcinoma as nongestational, as the genotype of tumor cells entirely corresponded with that of oral mucosal cells. BEP therapy and chemotherapy regimens administered for nongestational choriocarcinoma and gestational choriocarcinoma proved ineffective, and the patient died 21 months after diagnosis of metastatic choriocarcinoma.

Conclusion: Metastaic nongestational pure choriocarcinoma of ovary is an extremely rare and an aggressive disease, frequently resulting in poor outcome.

\section{Background}

Pure ovarian choriocarcinoma can be classified into two groups based on origin: gestational or nongestational. The gestational type presents as either a metastasis of uterine choriocarcinoma, or as primary choriocarcinoma following ovarian pregnancy, including hydatidiform mole $(1-3)$. The nongestational type emerges as a malignant germ cell tumor, but is extremely rare because most malignant germ cell tumors are mixed-type and consist of various malignant components, including immature teratoma, dysgerminoma, yolk sac tumor, and choriocarcinoma(4-6). Diagnosing pure ovarian choriocarcinoma as gestational or nongestational is essential, because the sensitivities to chemotherapy and prognosis of these two types appear to differ despite the identical pathological findings(7, 8). DNA short tandem repeat (STR) analysis is a critical modality to differentiate the types independent of common clinical information, such as medical history and/or imaging findings. Herein, we report a case of metastatic primary pure ovarian choriocarcinoma identified as nongestational by DNA STR analysis 
that showed chemo-resistance to regimens administered for gestational choriocarcinoma as well as nongestational choriocarcinoma with a review of the literature.

\section{Case Presentation}

A 38-year old nulliparous woman experienced headache, nausea, and a defect in the visual field over a period of days. Computed tomography (CT) and magnetic resonance imaging (MRI) of the head detected a tumor with midline shift in the left occipital lobe, $4 \mathrm{~cm}$ in diameter (Fig. 1a). As a result, she had undergone resection of the brain tumor. Histological examination of the resected specimen confirmed pure choriocarcinoma. Serum concentration of human chorionic gonadotropin (hCG) was $5030 \mathrm{IU} / \mathrm{L}$. Serum carbohydrate antigen 125 and a-fetoprotein levels were within normal range. Abdominal and pelvic CT and MRI demonstrated a solid left ovarian tumor, $5.5 \mathrm{~cm}$ in diameter (Fig. 1b). However, no evidence of tumor in the uterus was seen. Chest CT revealed metastatic choriocarcinoma in the left lung (Fig. 1C). Consequently, her disease was diagnosed as primary pure ovarian choriocarcinoma and two cycles of a combination chemotherapy regimen comprising methotrexate, etoposide, and actinomycin-D (MEA therapy) which is commonly used for gestational choriocarcinoma, were administered. However, each administration of this regimen caused strong bone marrow suppression and a new metastasis appeared in the cerebellum after the second cycle of MEA therapy. Therefore, she was referred to our hospital.

The third cycle of MEA therapy was administered with concomitant administration of pegfilgrastim to avoid bone marrow suppression. Gamma knife therapy was also performed for the cerebellar metastatic lesions. However, hCG levels increased continuously and the chemotherapy regimen was changed to a combination chemotherapy regimen of bleomycin, etoposide, and cisplatin (BEP therapy), which is commonly used for nongestational choriocarcinoma (malignant germ cell tumor). Two cycles of BEP therapy were effective and total abdominal hysterectomy was performed with bilateral salpingooophorectomy and segmentectomy of the left lung. Histologic examination of the left ovarian tumor demonstrated vast central necrosis and remnant marginal choriocarcinoma with no evidence of other germ cell elements, leading to a diagnosis of pure choriocarcinoma of the ovary (Fig. 2). BEP therapy and surgical resection achieved the nadir level of hCG (2.2 IU/L) (Fig. 3). However, two additional cycles of BEP therapy failed to achieve complete remission, and hCG level increased steeply when chest CT revealed multiple metastases in the lungs.

Her clinical history was reevaluated to identify the origin of choriocarcinoma and choose an appropriate chemotherapy regimen. She was unmarried, but had had sexual intercourse with her partner. Her menstrual cycle had been irregular since the time she had suffered subarachnoid hemorrhage due to rupture of an aneurysm on the posterior inferior cerebellar artery 10 months before development of the present illness. Therefore, the possibility of gestational choriocarcinoma subsequent to unrecognized pregnancy could not be ruled out. Consequently, we performed DNA STR analysis using a capillary electrophoresis system. Written informed consent was obtained from the patient for DNA extraction and DNA STR analysis, and this investigation was approved by the Ethics Committee of Nagoya University 
Graduate School of Medicine. DNA was extracted from oral mucosal cells of the patient and frozen cancer tissue, using a QIAamp® DNA Micro kit (Qiagen, Hilden, Germany) according to the protocol provided by the manufacturer. One microliter of extracted DNA was amplified using an AmpFeSTR® Identifiler ${ }^{\circledR}$ Plus PCR Amplification Kit (Life Technologies, Waltham MA) for 15 STR loci and the amelogenin locus for gender determination, for a total of 16 DNA markers, as previously described $(9,10)$. One microliter of amplified DNA was capillary electrophoresed on an Applied Biosystems 3130xl Genetic Analyzer (Life Technologies), and the detected peaks were genotyped automatically using GeneMapperID software version 3.2.1 (Life Technologies) as previously described $(9,10)$. Part of the STR analysis is shown in Fig. 4. Genomic DNA alleles of the tumor were entirely identical to those of the patient, confirming that the choriocarcinoma was nongestational.

A combination chemotherapy regimen of MEA, paclitaxel and cisplatin (TP), fluorouracil and actinomycin-D (FA), ifosfamide, cisplatin, and etoposide (ICE), etoposide, methotrexate, actinomycin$\mathrm{D} /$ cyclophosphamide and vincristine (EMA/CO) with segmentectomy of the right lung were administered after tumor relapse, but no chemotherapy regimens successfully suppressed tumor activity. The patient died 21 months after the diagnosis of choriocarcinoma.

\section{Discussion And Conclusions}

In this report, we have presented a case of metastatic pure ovarian nongestational choriocarcinoma that was differentiated from gestational choriocarcinoma by DNA STR analysis. This case showed resistance to chemotherapy regimens commonly administered for malignant germ cell tumor (nongestational choriocarcinoma) and for gestational choriocarcinoma.

When histological examination reveals pure ovarian choriocarcinoma lacking uterine lesions, as in this case, the nongestational is often presumed without careful attention, because gestational choriocarcinoma commonly originates from the uterus. Indeed, gestational choriocarcinoma without uterine lesions on initial presentation is very rare(11). However, one paper reported a case of gestational choriocarcinoma (confirmed by DNA analysis) with renal and pulmonary metastases without any uterine lesions(12). This may be due to spontaneous regression of the uterine tumor, possibly because gestational choriocarcinoma is a tissue allograft for the patient that induces strong immune reactions from the patient. In addition, not a few case reports have described primary ovarian gestational choriocarcinoma following ovarian pregnancy including hydatidiform mole(1-3). In those clinical settings, uterine lesions are not necessarily present. Accordingly, precise differentiation of gestational and pure nongestational choriocarcinoma based on histological examination or lesion spread is impractical. A history of past pregnancies is thus crucial. However, even with such information, determining whether the choriocarcinoma is gestational or nongestational is difficult based on the pregnancy history alone, except in cases of patients who are sexually immature, have never had sexual intercourse, or are unable to conceive, because not a few spontaneous abortions pass unrecognized. In the present case, the patient had a fixed partner, had engaged in regular sexual intercourse, and had irregular menstruation cycles 
before the development of the disease, meaning that the possibility of gestational choriocarcinoma due to unrecognized abortions could not be ruled out.

A STR is a microsatellite consisting of a unit of 2-10 nucleotides repeated several to hundreds of times, and STR analysis allows evaluation of the highly polymorphic STR loci where each individual shows differing numbers of repeated sequences of nucleotides. DNA STR analysis is frequently used in forensic genetics (13) and population genetics(14) to match DNA profiles to an individual. Recently, STR analysis has been utilized to clarify the origin of choriocarcinoma and to differentiate gestational and pure nongestational choriocarcinoma when definitive evidence has not been forthcoming from pathological or other clinical findings $(7,15-20)$. Because the genome in nongestational choriocarcinoma comprises only a maternal (patient) allele, whereas gestational choriocarcinoma contains a paternal allele, DNA STR analysis can precisely distinguish these two types of choriocarcinoma. In the present case, the tumor genotype entirely matched that of the patient, confirming the nongestational type.

Nongestational pure choriocarcinoma of ovary is an extremely rare, especially with metastatic disease (Table 1) (21-27), .The prognosis of pure nongestational choriocarcinoma is widely considered to be worse than that of gestational choriocarcinoma despite these two types of choriocarcinoma being pathologically identical. If this is indeed the case, such differences may be because the genome of gestational choriocarcinoma is totally or partially different from that of the patient, whereas nongestational choriocarcinoma is genetically identical to the patient, leading to differences in host immunoreactions. However, given with rarity of these pathological entities, no reports have analyzed large numbers of choriocarcinoma cases and compared prognoses between types as diagnosed based on DNA analyses. Combination chemotherapy regimens employing methotrexate, etoposide, and actinomycin-D such as EMA/CO or MEA are administered as first-line therapies against gestational choriocarcinoma, and the complete response rate for these chemotherapy regimens against this disease exceeds $70 \%(28)$. Regimens including cisplatin such as TP and BEP therapies are applied for resistant or relapsed disease, with an overall survival rate with or without adjuvant radiotherapy or surgery of around $90 \%(29)$. Nongestational choriocarcinoma usually develops as a component of mixed-type germ cell tumor, and seems to show favorable prognosis following surgery and BEP regimen $(30,31)$. When nongestational choriocarcinoma emerges as a pure type, however, the tumor behavior seems more aggressive. Cisplatin regimens are usually used for resistant or relapsed disease, as well as for gestational choriocarcinoma. In the present case, the tumor was confirmed as pure nongestational choriocarcinoma by DNA STR analysis, but showed multiple chemo-resistances against chemotherapy regimens typically administered against both nongestational or gestational choriocarcinoma.

We have presented a case of metastatic pure nongestational choriocarcinoma that showed poor sensitivity to chemotherapy and the death of the patient. Precise differentiation of gestational and nongestational choriocarcinoma based on STR analysis independent of common clinical information such as the medical history and/or imaging findings is essential for determining appropriate chemotherapy regimens and evaluating the prognosis. With distant metastasis, pure ovarian nongestational choriocarcinoma is highly malignancy and the prognosis is poor. 


\section{List Of Abbreviations}

STR, short tandem repeat; CT, computed tomography; MRI, magnetic resonance imaging; hCG, human chorionic gonadotropin; MEA, methotrexate, etoposide, and actinomycin-D; BEP, bleomycin, etoposide, and cisplatin; TP, paclitaxel and cisplatin; FA, fluorouracil and actinomycin-D; mini ICE, ifosfamide, cisplatin, and etoposide; EMA/CO, etoposide, methotrexate, actinomycin-D/cyclophosphamide and vincristine.

\section{Declarations}

\section{Ethics approval and consent to participate}

This study was conducted in accordance with the Declaration of Helsinki, and the use of data/material only for research purposes. Approval was obtained from the Ethics Committee of Nagoya University Graduate School of Medicine (reference number 2017-0325-11414). Written informed consent was obtained from the patient about participation in STR analysis.

\section{Consent for publication}

Written informed consent was obtained from the patient about publication of this case report.

\section{Availability of data and materials}

The datasets generated and/or analyzed during the current study are not publicly available due to individual privacy, but are available from the corresponding author on reasonable request

\section{Competing interests}

The authors declare that they have no competing interests.

\section{Funding}

This work was supported by a research grant from the Aichi Cancer Research Foundation.

\section{Authors' contributions}

$\mathrm{KiN}, \mathrm{YI}$, and $\mathrm{KaN}$ acquired the medical report and data from the patient. KiN, EY, and TY performed STR analysis and interpretation of the data. KiN drafted the case report section. EY and HK revised the final manuscript. All authors read and approved the final paper.

\section{Acknowledgements}

None

\section{References}


1. Lorigan PC, Grierson AJ, Goepel JR, Coleman RE, Goyns MH. Gestational choriocarcinoma of the ovary diagnosed by analysis of tumour DNA. Cancer letters. 1996;104(1):27-30.

2. Namba A, Nakagawa S, Nakamura N, Takazawa Y, Kugu K, Tsutsumi O, et al. Ovarian choriocarcinoma arising from partial mole as evidenced by deoxyribonucleic acid microsatellite analysis. Obstetrics and gynecology. 2003;102(5 Pt 1):991-4.

3. Joneborg U, Papadogiannakis N, Lindell G, Marions L. Choriocarcinoma following ovarian hydatidiform mole: a case report. The Journal of reproductive medicine. 2011;56(11-12):511-4.

4. Kurman RJ, Norris HJ. Malignant mixed germ cell tumors of the ovary. A clinical and pathologic analysis of 30 cases. Obstetrics and gynecology. 1976;48(5):579-89.

5. Vance RP, Geisinger KR. Pure nongestational choriocarcinoma of the ovary. Report of a case. Cancer. 1985;56(9):2321-5.

6. Raju GC, Woo J, Marchack D, Naraynsingh V. Primary nongestational choriocarcinoma of the ovary. Postgraduate medical journal. 1985;61(718):757-8.

7. Savage J, Adams E, Veras E, Murphy KM, Ronnett BM. Choriocarcinoma in Women: Analysis of a Case Series With Genotyping. The American journal of surgical pathology. 2017;41(12):1593-606.

8. Zhao J, Xiang Y, Wan XR, Feng FZ, Cui QC, Yang XY. Molecular genetic analyses of choriocarcinoma. Placenta. 2009;30(9):816-20.

9. Yamamoto E, Niimi K, Shinjo K, Yamamoto T, Fukunaga M, Kikkawa F. Identification of causative pregnancy of gestational trophoblastic neoplasia diagnosed during pregnancy by short tandem repeat analysis. Gynecologic oncology case reports. 2014;9:3-6.

10. Fujii K, Yamashita Y, Yamamoto T, Takahashi K, Hashimoto K, Miyata T, et al. Ovarian mucinous tumors arising from mature cystic teratomas--a molecular genetic approach for understanding the cellular origin. Human pathology. 2014;45(4):717-24.

11. Seckl MJ, Sebire NJ, Berkowitz RS. Gestational trophoblastic disease. Lancet (London, England). 2010;376(9742):717-29.

12. Li HM, Hou WC, Lai YJ, Kao CC, Chao TK, Yu MH, et al. Gestational choriocarcinoma with renal and pulmonary metastases lacking a primary uterine origin. Taiwanese journal of obstetrics \& gynecology. 2016;55(6):881-5.

13. Gill P, Haned H, Bleka O, Hansson O, Dorum G, Egeland T. Genotyping and interpretation of STR-DNA: Low-template, mixtures and database matches-Twenty years of research and development. Forensic science international Genetics. 2015;18:100-17.

14. Zhang DX, Hewitt GM. Nuclear DNA analyses in genetic studies of populations: practice, problems and prospects. Molecular ecology. 2003;12(3):563-84.

15. Aranake-Chrisinger J, Huettner PC, Hagemann AR, Pfeifer JD. Use of short tandem repeat analysis in unusual presentations of trophoblastic tumors and their mimics. Human pathology. 2016;52:92-100.

16. Wang $Y$, Yang $Y$, Teng F, Zhang H, Xue F. Pure nongestational uterine choriocarcinoma in a postmenopausal Chinese woman confirmed with short tandem repeat analysis. American journal of 
obstetrics and gynecology. 2014;211(1):e1-3.

17. Hirata $Y$, Yanaihara N, Yanagida S, Fukui K, Iwadate K, Kiyokawa T, et al. Molecular genetic analysis of nongestational choriocarcinoma in a postmenopausal woman: a case report and literature review. International journal of gynecological pathology : official journal of the International Society of Gynecological Pathologists. 2012;31(4):364-8.

18. Nakayama M, Namba A, Yasuda M, Hara M, Ishihara O, Itakura A. Gestational choriocarcinoma of Fallopian tube diagnosed with a combination of p57KIP2 immunostaining and short tandem repeat analysis: case report. The journal of obstetrics and gynaecology research. 2011;37(10):1493-6.

19. Yamamoto E, Ino K, Yamamoto T, Sumigama S, Nawa A, Nomura S, et al. A pure nongestational choriocarcinoma of the ovary diagnosed with short tandem repeat analysis: case report and review of the literature. International journal of gynecological cancer : official journal of the International Gynecological Cancer Society. 2007;17(1):254-8.

20. Koo HL, Choi J, Kim KR, Kim JH. Pure non-gestational choriocarcinoma of the ovary diagnosed by DNA polymorphism analysis. Pathology international. 2006;56(10):613-6.

21. Stevens RP, Peirce TH, Dooley PC, Parsons GH. Bilateral spontaneous pneumothorax in nongestational choriocarcinoma. Can Med Assoc J. 1979;120(7):830-1.

22. Raju GC, Woo J, Marchack D, Naraynsingh V. Primary nongestational choriocarcinoma of the ovary. Postgraduate medical journal. 1985;61(718):757-8.

23. Tsujioka $H$, Hamada $H$, Miyakawa $T$, Hachisuga $T$, Kawarabayashi T. A pure nongestational choriocarcinoma of the ovary diagnosed with DNA polymorphism analysis. Gynecologic oncology. 2003;89(3):540-2.

24. Park SH, Park A, Kim JY, Kwon JH, Koh SB. A case of non-gestational choriocarcinoma arising in the ovary of a postmenopausal woman. J Gynecol Oncol. 2009;20(3):192-4.

25. Gremeau AS, Bourdel N, Kondo W, Jardon K, Canis M. Management of non-gestational ovarian choriocarcinoma: laparoscopy can be essential. Report of two cases. Eur J Obstet Gynecol Reprod Biol. 2010;152(1):113-4.

26. Exman P, Takahashi TK, Gattás GF, Cantagalli VD, Anton C, Nalesso F, et al. Primary ovary choriocarcinoma: individual DNA polymorphic analysis as a strategy to confirm diagnosis and treatment. Rare Tumors. 2013;5(2):89-92.

27. Rao KVLN, Konar S, Gangadharan J, Vikas V, Sampath S. A pure non-gestational ovarian choriocarcinoma with delayed solitary brain metastases: Case report and review of the literature. $J$ Neurosci Rural Pract. 2015;6(4):578-81.

28. Lurain JR. Gestational trophoblastic disease II: classification and management of gestational trophoblastic neoplasia. American journal of obstetrics and gynecology. 2011;204(1):11-8.

29. Brown J, Naumann RW, Seckl MJ, Schink J. 15years of progress in gestational trophoblastic disease: Scoring, standardization, and salvage. Gynecologic oncology. 2017;144(1):200-7.

30. Gershenson DM, Morris M, Cangir A, Kavanagh JJ, Stringer CA, Edwards CL, et al. Treatment of malignant germ cell tumors of the ovary with bleomycin, etoposide, and cisplatin. Journal of clinical 
oncology : official journal of the American Society of Clinical Oncology. 1990;8(4):715-20.

31. Williams S, Blessing JA, Liao SY, Ball H, Hanjani P. Adjuvant therapy of ovarian germ cell tumors with cisplatin, etoposide, and bleomycin: a trial of the Gynecologic Oncology Group. Journal of clinical oncology : official journal of the American Society of Clinical Oncology. 1994;12(4):701-6.

\section{Tables}

Due to technical limitations, table 1 is only available as a download in the Supplemental Files section.

\section{Figures}

(a)

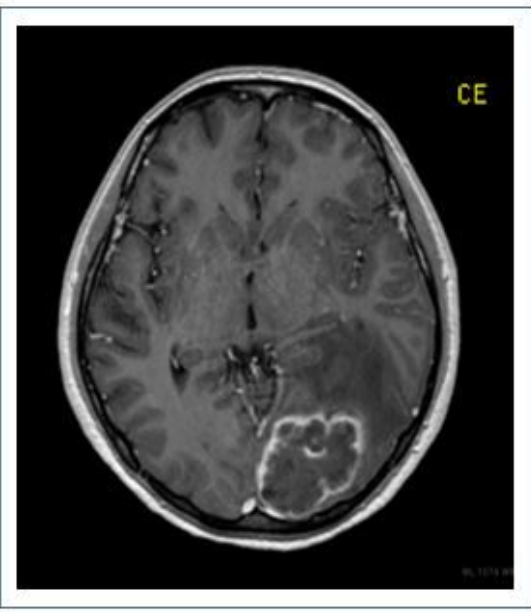

(b)

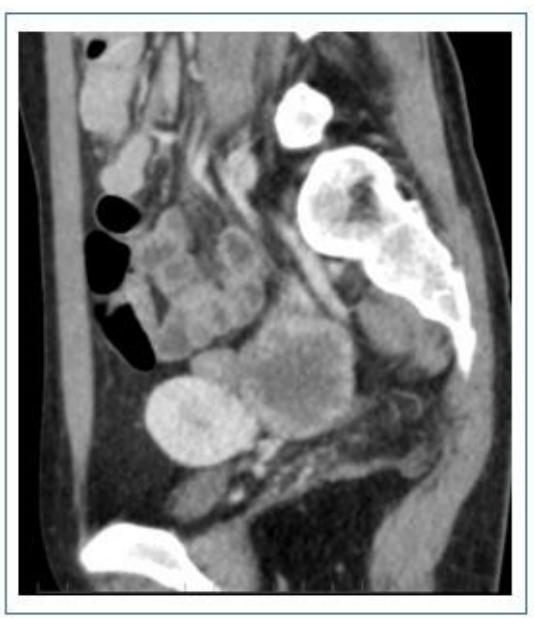

(C)

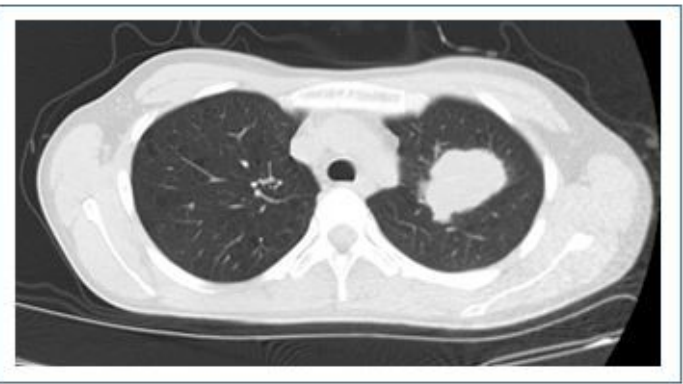

Figure 1

(a). Magnetic resonance imaging of the brain Findings from contrast-enhanced T1-weighted magnetic resonance imaging of the brain. A tumor ( $4 \mathrm{~cm}$ in diameter) is seen causing midline shift in the left occipital lobe. The ridge of the tumor is enhanced. (b). Contrast-enhanced CT of the abdomen and pelvis Contrast-enhanced CT of the abdomen and pelvis, showing a solid left ovarian tumor $(5.5 \mathrm{~cm}$ in diameter) and enhanced with a ridge. (c). Chest CT Chest CT reveals metastatic choriocarcinoma in the left lung. 

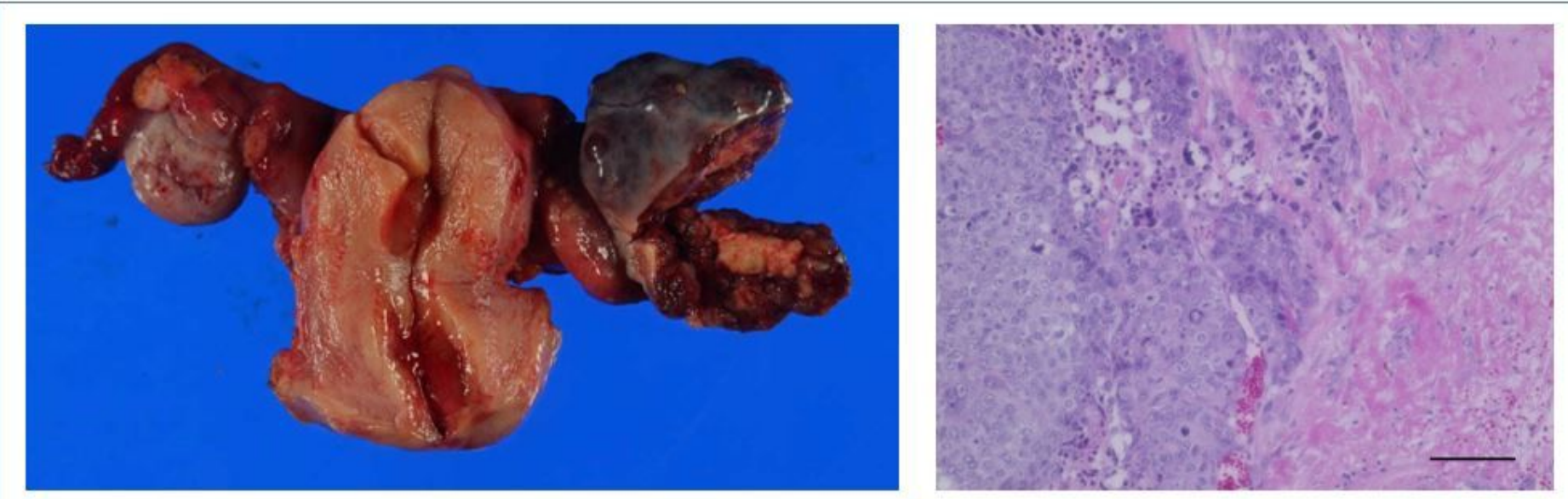

Figure 2

Macroscopic and microscopic findings Macroscopic findings of the left ovarian tumor, showing massive central necrosis (left). Microscopic appearance of the tumor shows atypical cytotrophoblastic and syncytiotrophoblastic cells and necrosis (right). Original magnification, $₫ 200$; scale bar, $500 \mu \mathrm{m}$.

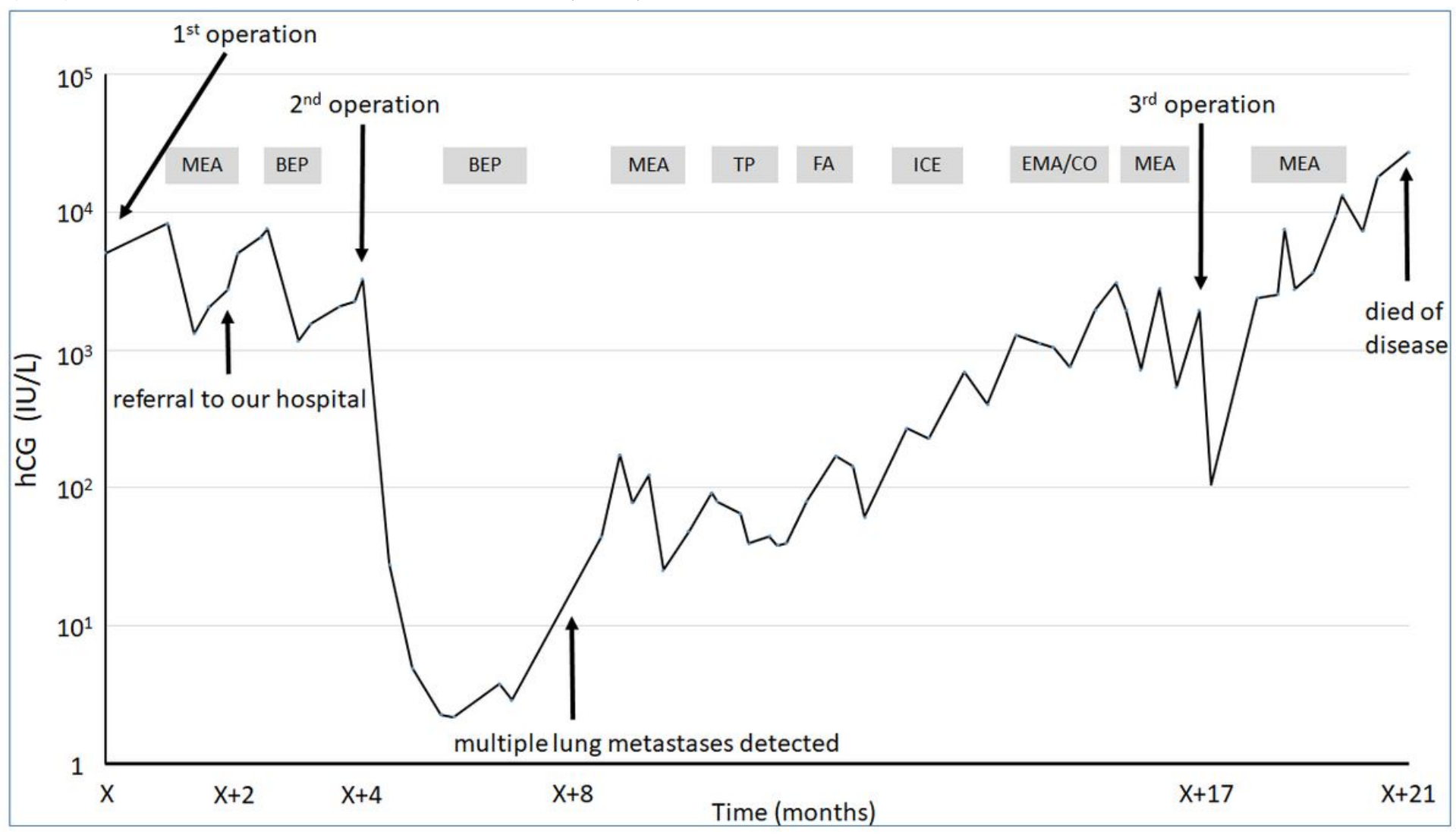

\section{Figure 3}

The trend of hCG 1st operation: Brain tumor resection, 2nd operation: Total abdominal hysterectomy with bilateral salpingo-oophorectomy and segmentectomy of left lung, 3rd operation: segmentectomy of right lung, MEA: methotrexate, etoposide, and actinomycin-D, BEP: bleomycin, etoposide, and cisplatin, TP: 
paclitaxel and cisplatin, FA: fluorouracil and actinomycin-D, ICE: ifosfamide, cisplatin, and etoposide, and EMA/CO: etoposide, methotrexate, actinomycin-D, cyclophosphamide and vincristine

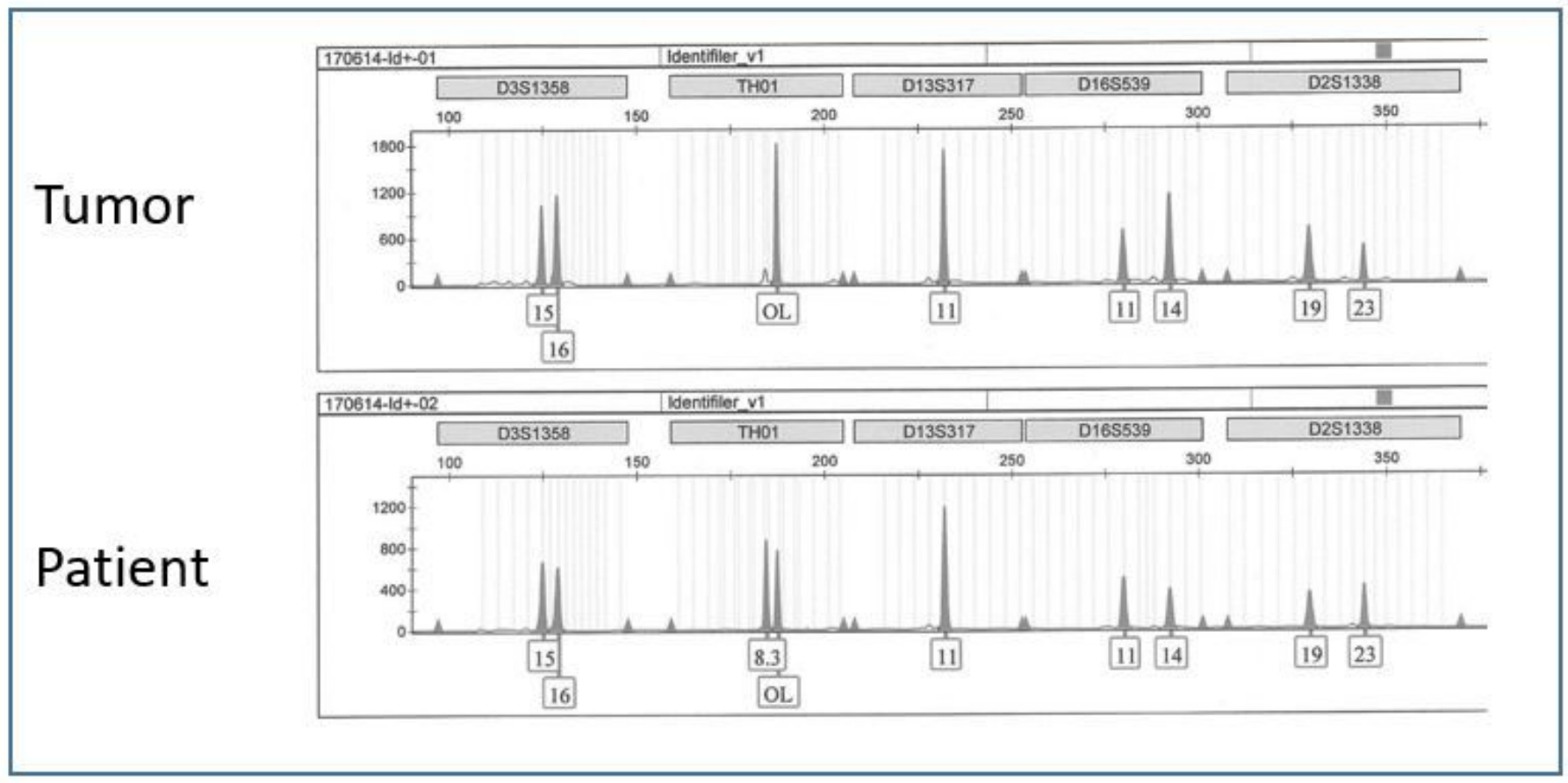

Figure 4

DNA STR analysis DNA STR analysis reveals the genomic DNA alleles of the tumor are identical to those of the patient, exhibiting that the choriocarcinoma is nongestational.

\section{Supplementary Files}

This is a list of supplementary files associated with this preprint. Click to download.

- Table1.xlsx 